\title{
Safety and efficacy of sintilimab combination therapy for the treatment of 48 patients with advanced malignant tumors
}

\author{
Nana Huang, Chenchen Zhao, Xueyang Hu, Congjun Zhang, Fuxing Xiong, Wei Huang, Liangshan Da, \\ Yuanyuan Shen, Hongyang $\mathrm{Wu}$
}

Department of Medical Oncology, First Affiliated Hospital of Anhui Medical University, Hefei, China

Contributions: (I) Conception and design: H Wu, N Huang; (II) Administrative support: $\mathrm{H}$ Wu; (III) Provision of study materials or patients: All authors; (IV) Collection and assembly of data: N Huang; (V) Data analysis and interpretation: N Huang; (VI) Manuscript writing: All authors; (VII) Final approval of manuscript: All authors.

Correspondence to: Hongyang Wu, MD, PhD. Department of Medical Oncology, First Affiliated Hospital of Anhui Medical University, Hefei 230031, China. Email: wuhoya1970@163.com.

Background: Sintilimab is a recombinant fully human anti-programmed death 1 (PD-1) monoclonal antibody that blocks the interaction of PD-1 with its ligand. We evaluated the safety and efficacy of sintilimab combined with chemotherapy and targeted therapy in the treatment of advanced malignant tumors.

Methods: We performed a retrospective analysis of the clinical data of patients with advanced malignant tumors treated with sintilimab combined with chemotherapy and targeted therapy admitted to the Third Ward of the Department of Medical Oncology, First Affiliated Hospital of Anhui Medical University, China, from July 2019 to February 2021. The objective response rate (ORR), disease control rate (DCR), progression-free survival (PFS), overall survival (OS) and related adverse reactions were analyzed.

Results: A total of 48 patients with advanced malignant tumors treated with sintilimab combined with chemotherapy and targeted therapy. All 48 patients completed 2 courses of treatment, and the ORR and DCR were $20.83 \%$ and $81.25 \%$. The median PFS for all patients in this study was 7 months, and the median OS was not yet reached. The median PFS for the first-line and second-line patients was 10 months, and the median OS was not yet reached. The median PFS for third-line and beyond patients was 7 months, and the median OS was 10 months. The differences in PFS and OS were both statistically significant. Adverse events occurred in 24 patients, of which 18 patients had grade I-II adverse events and 6 patients had grade III-IV adverse events.

Conclusions: Sintilimab is an inexpensive PD-1 drug produced in China. Sintilimab combination therapy showed good safety in the treatment of advanced malignant tumors, with increases in the treatment efficacy and DCR for advanced tumors. Because of few adverse reactions and proven efficacy, sintilimab combination therapy can be used as an option for the treatment of advanced malignant tumors.

Keywords: Sintilimab; advanced malignant tumors; objective response rate (ORR); disease control rate (DCR); adverse reactions

Submitted Sep 22, 2021. Accepted for publication Jan 17, 2022.

doi: $10.21037 /$ tcr-22-54

View this article at: https://dx.doi.org/10.21037/tcr-22-54

\section{Introduction}

In recent years, the incidence of malignant tumors worldwide has increased year to year, seriously affecting human health. The treatment of tumors has also progressed from approaches involving surgery, radiotherapy, and chemotherapy to precision targeted therapy, entering a new era of immunotherapy. The combination of classical tumor treatment methods and immune checkpoint inhibitors (ICIs) effectively improves the quality of life of tumor patients and prolongs the survival time of patients with advanced 
tumors $(1,2)$. Immune checkpoint molecules mainly include programmed death 1 (PD-1)/programmed death ligand 1 (PD-L1) and cytotoxic T lymphocyte-associated antigen 4 (CTLA-4) $(3,4)$. PD-1 is an important immunosuppressive molecule and a member of the CD28 superfamily (5). PD-1/ PD-L1 inhibitors can block the binding of PD-1 and PDL1, block negative regulatory signals, restore the activity of $\mathrm{T}$ cells, and enhance antitumor immune responses, thereby achieving an antitumor effect $(6,7)$. PD-1 and PD-L1 inhibitors have shown significant efficacy in the treatment of a variety of advanced malignant tumors. Breakthroughs in the treatment of non-small cell lung cancer (NSCLC), esophageal cancer and microsatellite instable tumors have been achieved, as represented by PD-1 monoclonal antibodies and PD-L1 monoclonal antibodies (8-10). As such, they have been approved as therapeutic options for a variety of malignant tumors. In recent years, ICIs have placed a spotlight on immunotherapy in tumor treatment. The gradual introduction of inexpensive PD-1 monoclonal antibodies made in China can benefit more Chinese patients with advanced cancer. Sintilimab is a PD-1 antibody made in China, and studies have shown that sintilimab has higher levels of PD-1 occupancy than nivolumab and pembrolizumab in peripheral blood mononuclear cells (PBMCs) and in mice injected with PBMCs. In patients, after a single intravenous infusion of sintilimab, a sustained occupancy of $\geq 95 \%$ of PD- 1 for 4 weeks was observed (11). In a variety of studies, this drug has shown good antitumor effects on various types of cancer.

Sintilimab combination therapy has been clinically tested in a variety of tumor treatments. In a multicenter, singlearm, phase II trial (ORIENT-1) that enrolled 96 adults with relapsed or refractory classical Hodgkin's lymphoma after 2 or more lines of chemotherapy, the objective response rate (ORR) was $80.4 \%$, and the disease control rate (DCR) was $97.8 \%$. Sintilimab has been approved for the treatment of relapsed or refractory classical Hodgkin's lymphoma because sintilimab is associated with a high remission rate and low toxicity $(12,13)$. Results from ORIENT-11 and ChiCTR-OIC-17013726 suggest that sintilimab has good safety and tolerability and has satisfactory efficacy in NSCLC $(14,15)$. Results from KEYNOTE-590 suggest that sintilimab also has good antitumor effects in esophageal cancer (16). Sintilimab has also been shown to be effective in the treatment of other tumors. The results of a clinical study regarding the efficacy of sintilimab in the treatment of advanced hepatocellular carcinoma were encouraging (17). In another retrospective single-center study involving 10 patients with advanced renal cell carcinoma, the ORR of sintilimab combined with axitinib was $40.00 \%$, and the DCR was $90.00 \%$ (18). The above results suggest that sintilimab may be a new therapeutic option.

In China, many patients with advanced malignancies typically have poor performance status and often do not respond to first- and second-line treatments. The clinical benefits of immunotherapy, chemotherapy, or targeted therapy alone are not obvious in these patients. Therefore, immune-based combination therapies have been developed to further increase the clinical response rates of patients with advanced malignancies. Many clinical studies have demonstrated the high efficacy and low toxicity of sintilimab; in addition, the price of sintilimab is more affordable and acceptable for Chinese patients. This study focuses on the clinical treatment of advanced tumor patients actually treated in Anhui, China. It is a real-world study. This study retrospectively analyzed the efficacy and safety of sintilimab combined with chemotherapy and targeted therapy in patients with advanced malignant tumors. The treatment results will better reflect the intuitive and real clinical effect of sintilimab combined with chemotherapy and targeted therapy in clinical treatment, it can provide a guidance and reference in the treatment of patients with advanced tumor in the future. We present the following article in accordance with the STROBE reporting checklist (available at https://tcr.amegroups.com/article/ view/10.21037/tcr-22-54/rc).

\section{Methods}

\section{General information}

The clinical data of patients with advanced malignant tumors who received sintilimab combined with chemotherapy and targeted drug treatment in the third ward of the Department of Oncology, First Affiliated Hospital of Anhui Medical University, China, from July 2019 to February 2021 were collected. Follow-up occurred until April 2021. As a retrospective study based on realworld data, our current study had certain limitations in the timeliness of the data and the number of cases, and thus we did not carry out analysis based on specific tumor subgroups. With larger sample size in the future, we will further explore whether the efficiency of sintilimab is higher in specific tumors than in other tumor types. All patients had a clear pathological diagnosis, with imaging indicating 
advanced tumors and Eastern Cooperative Oncology Group (ECOG) scores ranging from 0 to 2 points. Patients with autoimmune system diseases and liver, lung or renal failure were excluded. The patients were expected to survive for more than half a year and signed informed consent forms for treatment. The drug withdrawal indications were treatment ineffectiveness, disease progression, intolerable toxicity, death, and abandonment of treatment. The study was conducted in accordance with the Declaration of Helsinki (as revised in 2013). The study was approved by institutional ethics board of First Affiliated Hospital of Anhui Medical University, Hefei, China (No. PJ2022-01-50) and individual consent for this retrospective analysis was waived.

\section{Treatment regimens}

All patients with advanced malignant tumors received sintilimab combination therapy. Patients received $200 \mathrm{mg}$ (Recommended dose in drug instructions) sintilimab intravenously over a period of 30-60 min, once every 3 weeks, until disease progression, death, unacceptable toxicity, or withdrawal of consent, for a maximum of 24 months. Chemotherapy and targeted drugs were administered in accordance with standard treatment regimens.

\section{Observation indicators}

Before each treatment with sintilimab, the patients underwent routine blood tests, biochemical tests, thyroid function tests, myocardial enzyme analysis, and electrocardiography. Imaging examinations were performed every 2 treatment courses to evaluate efficacy, and adverse reactions during treatment were recorded in detail.

\section{Evaluation of efficacy and adverse reactions}

In accordance with RECIST 1.1, efficacy was evaluated and classified as complete response (CR), partial response $(\mathrm{PR})$, stable disease (SD) and progressive disease (PD). $\mathrm{ORR}=(\mathrm{CR}+\mathrm{PR}) /$ total number of cases $\times 100 \%$, and DCR $=(\mathrm{CR}+\mathrm{PR}+\mathrm{SD}) /$ total number of cases $\times 100 \%$. Adverse reactions were evaluated in accordance with the Common Terminology Criteria for Adverse Events (CTCAE) 5.0.

\section{Statistical analysis}

Disease control was calculated as the proportion of patients with a best overall response of complete remission, partial remission, or stable disease. Time to response was defined as the time from the first treatment administration to the first incidence of treatment response (either complete remission or partial remission). Progression-free survival was defined as the time from first treatment administration to disease progression or death from any cause, whichever came first.

Kaplan-Meier survival curves were plotted using GraphPad Prism 8.3 (GraphPad Software Inc), with involvement of proportional hazard model and the Log rank test. $\mathrm{P}<0.05$ was considered statistically significant.

\section{Results}

\section{Clinical characteristics}

From July 2019 to February 2021, a total of 48 patients (35 males and 13 females) were included in this retrospective analysis. The patients were 28-81 years old, with a median age of 59 years. Among the tumors, there were 3 cases of head and neck squamous cell carcinoma, 11 cases of esophageal cancer, 12 cases of gastric cancer, 2 cases of intestinal cancer, 2 cases of liver cancer, 1 case of gallbladder cancer, 2 cases of malignant melanoma, 2 cases of urothelial cancer, 7 cases of lung cancer, 2 cases of malignant lymphoma, 1 case of ovarian cancer, 1 case of soft tissue sarcoma, and 2 cases of breast cancer. The baseline characteristics of the patients are summarized in Table 1.

\section{Treatment regimens}

In this study, a total of 48 patients received sintilimab combined with chemotherapy. The chemotherapy regimen was selected based on the tumor type. The main chemotherapeutic drugs involved included gemcitabine, oxaliplatin, capecitabine, irinotecan, nab-paclitaxel, tegafur, and nedaplatin. Two patients received targeted drugs combined with anlotinib, 1 patient received a targeted drug combined with apatinib mesylate, and 1 patient received a targeted drug combined with sorafenib.

\section{Efficacy assessment}

All patients were treated with sintilimab for 2 courses or more. The ORR and DCR increased as the number of courses of sintilimab combined with chemotherapy increased. The ORR and DCR for the 2 courses of 
Table 1 Clinical characteristics of patients with advanced tumors

\begin{tabular}{|c|c|}
\hline Clinical characteristics of the patients & Number of patients (\%) \\
\hline \multicolumn{2}{|l|}{ Sex } \\
\hline Male & 35 (72.92) \\
\hline Female & $13(27.08)$ \\
\hline \multicolumn{2}{|l|}{ ECOG score } \\
\hline $0-1$ & $40(83.33)$ \\
\hline $2-3$ & $8(16.67)$ \\
\hline \multicolumn{2}{|l|}{ Age } \\
\hline$\geq 60$ years old & $24(50.00)$ \\
\hline$<60$ years old & $24(50.00)$ \\
\hline \multicolumn{2}{|l|}{ Tumor type } \\
\hline Soft tissue sarcoma & $1(2.08)$ \\
\hline Intestinal cancer & $2(4.17)$ \\
\hline Esophageal cancer & $11(22.92)$ \\
\hline Malignant lymphoma & $2(4.17)$ \\
\hline Gastric cancer & $12(25.00)$ \\
\hline Breast cancer & $2(4.17)$ \\
\hline Liver cancer & $2(4.17)$ \\
\hline Urothelial carcinoma & $2(4.17)$ \\
\hline Ovarian cancer & $1(2.08)$ \\
\hline Lung cancer & $7(14.58)$ \\
\hline Malignant melanoma & $2(4.17)$ \\
\hline Head and neck squamous cell carcinoma & $3(6.25)$ \\
\hline Gallbladder cancer & $1(2.08)$ \\
\hline \multicolumn{2}{|l|}{ Treatment regimen } \\
\hline Sintilimab combined with chemotherapy & $44(91.67)$ \\
\hline Sintilimab combined with apatinib & $1(2.08)$ \\
\hline Sintilimab combined with anlotinib & $2(4.17)$ \\
\hline Sintilimab combined with sorafenib & $1(2.08)$ \\
\hline \multicolumn{2}{|l|}{ Treatment line } \\
\hline First and second line & 35 (72.92) \\
\hline Third line and beyond & $13(27.08)$ \\
\hline \multicolumn{2}{|l|}{ Treatment course } \\
\hline 2 courses or more & $48(100.00)$ \\
\hline 4 courses or more & 27 (56.25) \\
\hline 6 courses or more & $11(22.92)$ \\
\hline 8 courses or more & $7(14.58)$ \\
\hline
\end{tabular}

treatment were $20.83 \%$ and $81.25 \%$, respectively. Twentyseven patients completed 4 or more courses; for these patients, the ORR and DCR were $44.44 \%$ and $81.48 \%$, respectively. Eleven patients completed 6 or more courses; for these patients, the ORR and DCR were $54.55 \%$ and $81.82 \%$, respectively. Seven patients completed 8 or more courses; for these patients, the ORR and DCR were $71.42 \%$ and $85.71 \%$, respectively (Table 2 ). One patient with advanced nasopharyngeal carcinoma, a 53-year-old male, had pulmonary metastases with rapid progression after 2 courses of second-line treatment. The lesions in the lungs basically disappeared, and the efficacy was significant after 2 courses of third-line treatment with sintilimab and gemcitabine combined with cisplatin chemotherapy. The imaging changes are shown in Figure 1.

\section{Progression-free survival (PFS)}

By the end of the follow-up period, disease progression or death had been reported for 24 patients. Four patients withdrew from the follow-up early, and their data were censored. The overall median PFS was 7 months (Figure 2A). The median PFS for the 35 patients who received first-line or second-line treatment was 10 months, and the median PFS for the patients who received thirdline treatment and beyond was 7 months, with a significant difference between the 2 groups $(\mathrm{P}<0.05)$. The efficacy for first-line and second-line patients was better than that for third-line and beyond patients (Figure 2B).

\section{Overall survival (OS)}

By the end of the follow-up period, 9 patients had died, and 35 patients were alive; 4 patients withdrew early from follow-up, and their data were censored. The overall median OS had not yet been reached (Figure $3 A$ ). Among the 35 patients who received first-line or second-line treatment, the median OS had not yet been reached. For the 13 patients who received third-line treatment and beyond, and the median OS was 10 months. The difference between the 2 groups was statistically significant $(\mathrm{P}<0.05)$ (Figure $3 B)$.

\section{Adverse reactions}

In this study, 24 patients had adverse reactions, including 18 cases of grade 1-2 reactions and 6 cases of grade 3 and above reactions. In the other 24 patients, there were no adverse reactions. The incidence of all grades of immune- 
Table 2 Evaluation of the efficacy of different treatment courses in patients with advanced tumors

\begin{tabular}{lcccccc}
\hline Treatment course & Total number of patients & CR & PR & SD & PD & ORR \\
\hline 2 courses & 48 & 0 & 10 & 29 & 9 & $20.83 \%$ \\
4 courses & 27 & 0 & 12 & 10 & 5 & $44.44 \%$ \\
6 courses & 11 & 0 & 6 & 3 & 2 & $54.55 \%$ \\
8 courses & 7 & 0 & 5 & 1 & 1 & $71.42 \%$ \\
\hline
\end{tabular}

CR, complete response; PR, partial response; SD, stable disease; PD, progressive disease; ORR, objective response rate; DCR, disease control rate; PR, partial response.
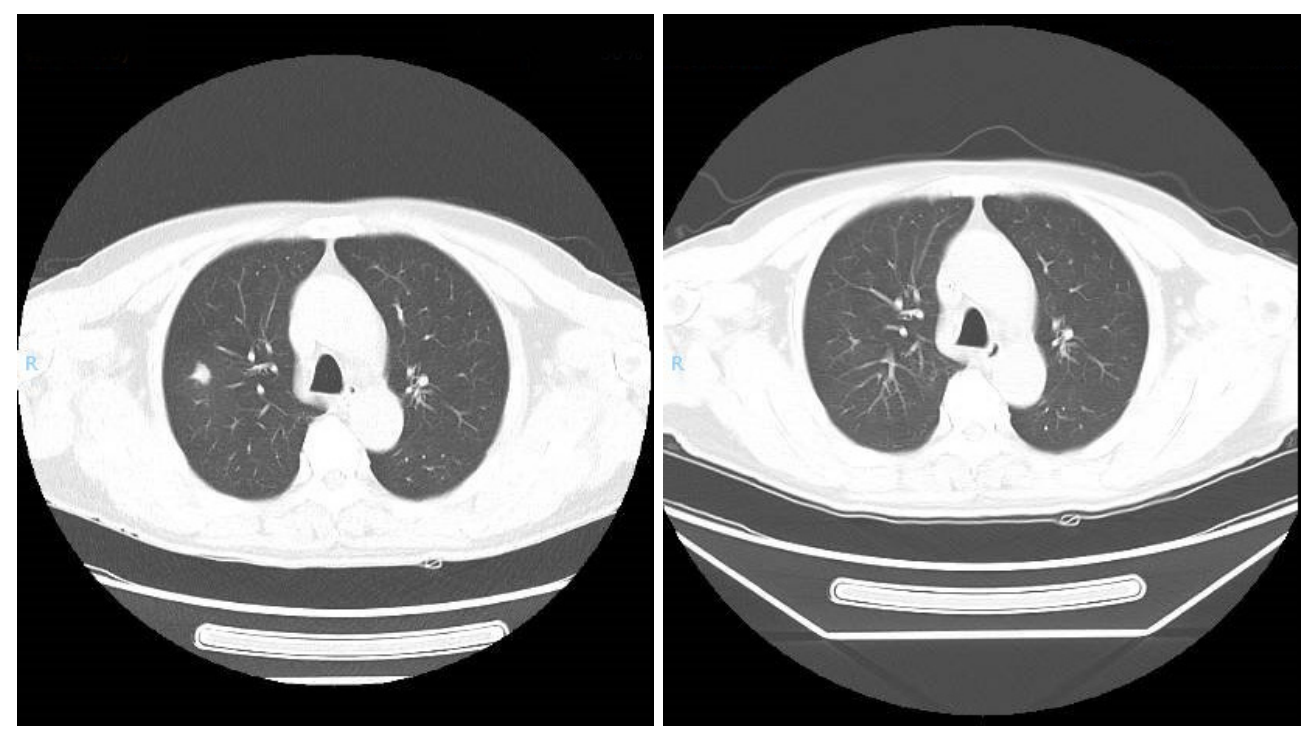

Figure 1 Nasopharyngeal carcinoma patient treated with sintilimab combination therapy; pulmonary lesions basically disappeared after 2 courses of treatment.
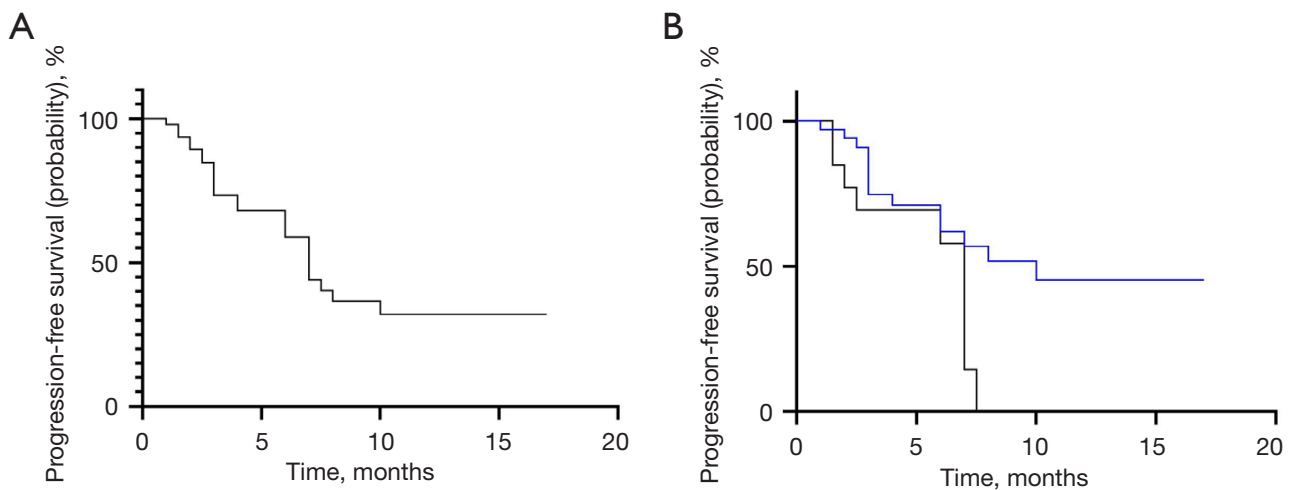

Figure 2 PFS of sintilimab in the treatment of advanced malignant tumors. (A) PFS of patients with advanced tumors who received sintilimab combination therapy. (B) The median PFS of first-line and second-line patients (blue line) was better than that of third-line and beyond patients (black line). PFS, progression-free survival. 

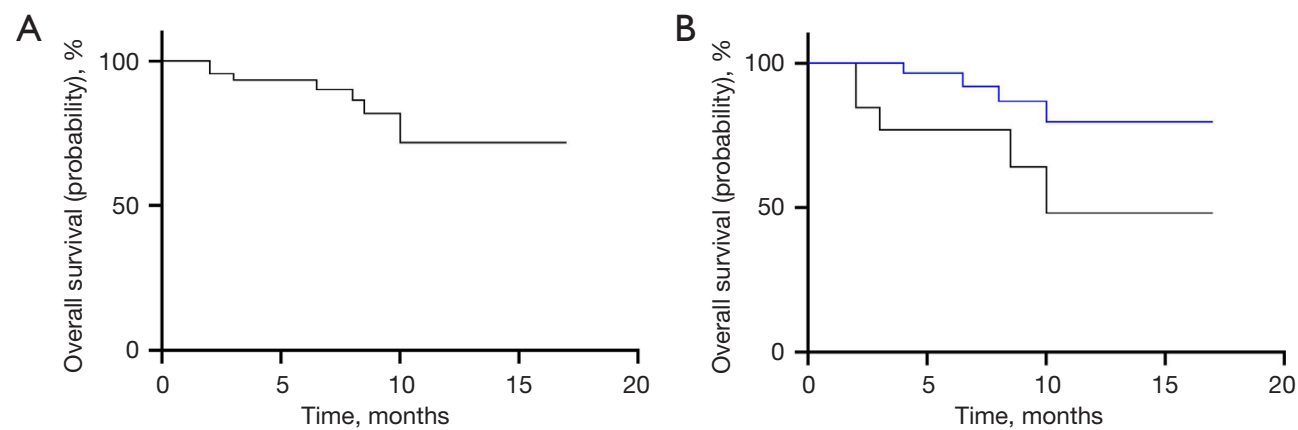

Figure 3 OS of sintilimab in the treatment of advanced malignant tumors. (A) OS of patients with advanced tumors who received sintilimab combination therapy; (B) The median OS of first-line and second-line patients (blue line) was better than that of third-line and beyond patients (black line). OS, overall survival.

Table 3 Adverse reactions related to immune combination therapy

\begin{tabular}{lccc}
\hline & & Patient number, No. (\%) & All levels \\
\cline { 2 - 4 } Adverse reactions & Grade 1-2 & Grade 3 and above & $24(50.00)$ \\
\hline Patients with adverse reactions & $18(37.50)$ & $6(12.50)$ & $2(4.17)$ \\
Skin reaction & $2(4.17)$ & - & $9(18.75)$ \\
Gastrointestinal reactions & $7(14.58)$ & $2(4.17)$ & $2(4.17)$ \\
Hypothyroidism & $2(4.17)$ & - & $4(8.33)$ \\
Liver damage & $3(6.25)$ & - & $1(2.08)$ \\
Immune-associated pneumonia & $1(2.08)$ & - & $1(2.08)$ \\
Immune-associated myocarditis & $1(2.08)$ & $2(4.17)$ & $7(14.58)$ \\
$\begin{array}{l}\text { Bone marrow suppression after } \\
\text { chemotherapy }\end{array}$ & $5(10.42)$ & $2(4.17)$ & $9(18.75)$ \\
Hypoproteinemia & $7(14.58)$ & - & $1(2.08)$ \\
Intestinal obstruction & $1(2.08)$ & - & \\
\hline
\end{tabular}

related toxicity was $50.00 \%$, the incidence of grade 1-2 toxic reactions was $37.50 \%$, and the incidence of grade 3 and above toxic reactions was $12.50 \%$. The most common adverse reactions were mainly grade 1 to 2 , for example, gastrointestinal reactions, bone marrow suppression after chemotherapy, rash with itching, immune-related thyroid dysfunction, abnormal liver function, immunerelated pneumonia, and immune-related myocarditis. Immunotherapy was permanently discontinued for 1 patient due to severe liver damage, and treatment was suspended for 1 patient due to immune-related myocarditis (Table 3).

\section{Discussion}

In this study, patients with a variety of advanced malignant tumors were enrolled. The median PFS after sintilimab combination treatment was 7 months. The median PFS for patients who received first-line and second-line regimens was 10 months, and the median OS had not yet been reached; these values were significantly better than those for patients who received third-line regimens and beyond and patients with high ECOG scores. Twelve patients were still using sintilimab at the end of the follow-up period.

Chemotherapy is a treatment that uses chemical drugs 

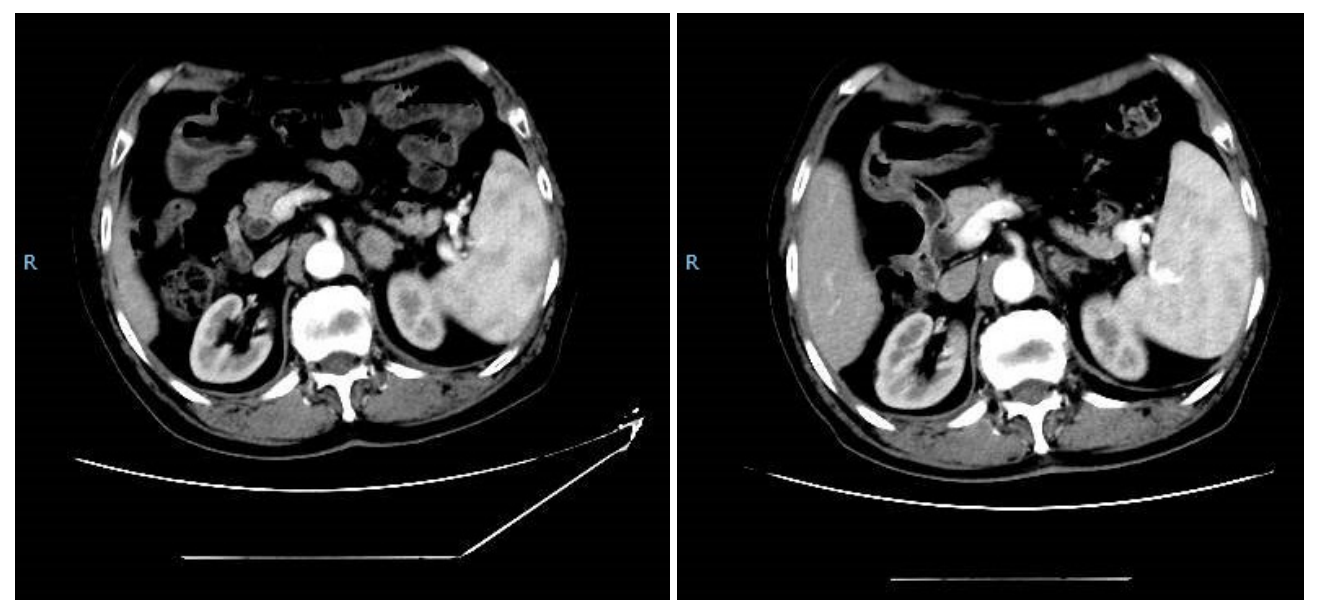

Figure 4 Lung squamous cell carcinoma (left) treated with 2 courses of second-line sintilimab combination therapy (right); the left adrenal lesion decreased significantly.

to prevent the proliferation, infiltration, and metastasis of cancer cells and to ultimately kill cancer cells. These drugs not only act on cancer cells but also affect normal cells in the body; furthermore, drug resistance can occur, limiting efficacy. Chemotherapy can induce the death of immunogenic tumor cells; therefore, immunotherapy may play a complementary role to chemotherapy. Results from KEYNOTE-590 (16) confirmed that the efficacy of immunotherapy combined with chemotherapy was superior to that of chemotherapy alone in advanced esophageal cancer. The KEYNOTE-189 (19), KEYNOTE-407 (20), and IMpower131 (21) clinical trials confirmed that in advanced NSCLC, the efficacy of immunotherapy combined with chemotherapy was superior to that of chemotherapy alone. In this study, the median PFS was 7 months, and the DCR was greater than $80 \%$, results similar to the efficacy achieved by immunotherapy combined with chemotherapy in the abovementioned studies.

In the KEYNOTE-189 clinical trial (19), the PFS for epidermal growth factor receptor (EGFR) mutationnegative or ALK-negative advanced NSCLC patients who received first-line pembrolizumab combined with pemetrexed and platinum was 8.8 months, a result similar to those reported for the ORIENT-11 trial. Data from the phase 3 CheckMate-649 trial (22) showed that the first-line combination therapy of nivolumab and chemotherapy showed a survival benefit in patients with advanced gastric cancer, in contrast to the chemotherapy alone. ORIENT-16 (23) demonstrated sintilimab in combination with chemotherapy significantly prolonged OS (18.4 months) in the first- line treatment of advanced gastric cancer, superior to that of nivolumab. In addition to its low price, sintilimab also had significantly lower toxicities than pembrolizumab and nivolumab. These results indicate that sintilimab may be an equivalent substitute for pembrolizumab or nivolumab in China for first-line PD-(L)1 combined with chemotherapy.

Many patients in this study showed significant remission after 2 courses of treatment. A 68-year-old male patient had right lung squamous cell carcinoma recurrence 8 months after surgery. The first-line treatment was sintilimab (200 mg) combined with nab-paclitaxel (400 mg). After 2 courses of treatment, an evaluation indicated a significant reduction in the metastases in the left adrenal gland and right kidney, a decrease in carcinoembryonic antigen (CEA) from 15.64 to $2.65 \mathrm{ng} / \mathrm{mL}$, PR, and a significant improvement in the patient's condition. The imaging changes are shown in Figure 4. We believe that sintilimab combination therapy has a significant effect in clinical practice.

Although sintilimab has shown effectiveness in the treatment of advanced malignant tumors, the potential side effects are still worthy of attention. In this study, the incidence of adverse events was $50.0 \%$ (24/48), and the incidence of grade 3 and above treatment-related adverse events (TRAEs) was $12.50 \%$ (6/48). There were no deaths caused by drug-related side effects. Among the adverse events, there were 4 cases of liver damage, with 3 cases that were grade 2 or less and 1 case that was grade 3 ; however, none of these 4 cases involved underlying liver disease. The 3 patients with grade 2 or less liver damage 
improved with symptomatic management and continued to receive sintilimab combination therapy. The patient with grade 3 liver damage was treated with hormones in combination with hepatoprotective therapy and gradually improved, without continuing treatment with sintilimab. In addition, among the adverse events, 1 case of myocarditis and 1 case of pneumonia occurred; although both may have been immune-related adverse events caused by sintilimab, their severity did not exceed grade 2 . The grade 3 adverse events were mainly gastrointestinal reactions, bone marrow suppression, and hypoproteinemia, all of which were considered to be chemotherapy-related side effects. The incidence and severity of adverse reactions to sintilimab combined with chemotherapy were basically consistent with those of chemotherapy drugs with known side effects. The addition of immunotherapy did increase the number of adverse events, and most adverse events were grade 1 to 2 . These results are consistent with those reported by Vincent K. Lam (24) in a study of sintilimab combined with chemotherapy in patients with lung cancer and are similar to those for NCT02937116 (25), which investigated sintilimab combined with chemotherapy in patients with gastric cancer. Compared with nivolumab or pembrolizumab, sintilimab is less toxic and has been proven to be safe (26-29).

This was a retrospective study with a small sample size. The results need to be further confirmed by largesample prospective clinical trials. Due to the diversity of combination treatment regimens, a comparative analysis between different regimens was not performed. In addition, the follow-up time was limited, and OS data were still being gathered. Initially, we planned to assess PD-(L)1 expression and efficacy. However, referring to relevant immunotherapeutic drugs in China, such as camrelizumab and toripalimab, we found that although PD-L1 expression was low or not detected, combined treatment still achieved good efficacy. A larger sample size would allow the detailed detection of immunotherapy-related markers, which may be beneficial for efficacy analyses. Our study data are based on observations of the clinical efficacy of sintilimab combination treatment in patients with advanced malignant tumors. The results of this study have certain guiding value for the future application of immunological drugs for the treatment of advanced malignant tumors.

\section{Conclusions}

Sintilimab combination therapy increases the treatment efficacy for patients with a variety of advanced tumors, and there is no significant increase in adverse events. The efficacy of sintilimab combination therapy for the treatment of more tumor types should be explored. In the future, sintilimab may be an important option for the clinical treatment of these advanced malignant tumors.

\section{Acknowledgments}

Funding: This study received funding from the project Phase II clinical Study of The Efficacy and Safety of A PD-1 Monoclonal Antibody Combined with Nab-Paclitaxel in The Second-Line Treatment of Patients with Advanced Esophageal and Gastric Junction Squamous Cell Carcinoma (No. 320.6750.2020-10-27, to Hongyang Wu).

\section{Footnote}

Reporting Checklist: The authors have completed the STROBE reporting checklist. Available at https://tcr. amegroups.com/article/view/10.21037/tcr-22-54/rc

Data Sharing Statement: Available at https://tcr.amegroups. com/article/view/10.21037/tcr-22-54/dss

Conflicts of Interest: All authors have completed the ICMJE uniform disclosure form (available at https://tcr. amegroups.com/article/view/10.21037/tcr-22-54/coif). HW received funding from the project Phase II clinical Study of The Efficacy and Safety of A PD-1 Monoclonal Antibody Combined with Nab-Paclitaxel in The SecondLine Treatment of Patients with Advanced Esophageal and Gastric Junction Squamous Cell Carcinoma (No. 320.6750.2020-10-27, to HW). The other authors have no conflicts of interest to declare.

Ethical Statement: The authors are accountable for all aspects of the work in ensuring that questions related to the accuracy or integrity of any part of the work are appropriately investigated and resolved. The study was conducted in accordance with the Declaration of Helsinki (as revised in 2013). The study was approved by institutional ethics board of First Affiliated Hospital of Anhui Medical University, Hefei, China (No. PJ2022-01-50) and individual consent for this retrospective analysis was waived.

Open Access Statement: This is an Open Access article distributed in accordance with the Creative Commons 
Attribution-NonCommercial-NoDerivs 4.0 International License (CC BY-NC-ND 4.0), which permits the noncommercial replication and distribution of the article with the strict proviso that no changes or edits are made and the original work is properly cited (including links to both the formal publication through the relevant DOI and the license). See: https://creativecommons.org/licenses/by-nc-nd/4.0/.

\section{References}

1. Chowdhury PS, Chamoto K, Honjo T. Combination therapy strategies for improving PD-1 blockade efficacy: a new era in cancer immunotherapy. J Intern Med 2018;283:110-20.

2. Fenwick C, Loredo-Varela JL, Joo V, et al. Tumor suppression of novel anti-PD-1 antibodies mediated through CD28 costimulatory pathway. J Exp Med 2019;216:1525-41.

3. Kennedy LB, Salama AKS. A review of cancer immunotherapy toxicity. CA Cancer J Clin 2020;70:86-104.

4. Marin-Acevedo JA, Dholaria B, Soyano AE, et al. Next generation of immune checkpoint therapy in cancer: new developments and challenges. J Hematol Oncol 2018;11:39.

5. $\mathrm{He} \mathrm{X}, \mathrm{Xu} \mathrm{C}$. Immune checkpoint signaling and cancer immunotherapy. Cell Res 2020;30:660-9.

6. Riley JL. PD-1 signaling in primary T cells. Immunol Rev 2009;229:114-25.

7. Wang $X$, Wang G, Wang Z, et al. PD-1-expressing B cells suppress CD4+ and CD8+ T cells via PD-1/PD-L1dependent pathway. Mol Immunol 2019;109:20-6.

8. Yang H, Wang K, Wang T, et al. The Combination Options and Predictive Biomarkers of PD-1/PDL1 Inhibitors in Esophageal Cancer. Front Oncol 2020;10:300.

9. Moya-Horno I, Viteri S, Karachaliou N, et al. Combination of immunotherapy with targeted therapies in advanced non-small cell lung cancer (NSCLC). Ther Adv Med Oncol 2018;10:1758834017745012.

10. Dudley JC, Lin MT, Le DT, et al. Microsatellite Instability as a Biomarker for PD-1 Blockade. Clin Cancer Res 2016;22:813-20.

11. Wang J, Fei K, Jing H, et al. Durable blockade of PD-1 signaling links preclinical efficacy of sintilimab to its clinical benefit. MAbs 2019;11:1443-51.

12. Shi Y, Su H, Song Y, et al. Safety and activity of sintilimab in patients with relapsed or refractory classical Hodgkin lymphoma (ORIENT-1): a multicentre, single-arm, phase
2 trial. Lancet Haematol 2019;6:e12-9.

13. Instructions for sintilimab injection (2020). Accessed October 4th. Available online: http://inn-qc.iqiaomai. com/5c89f09e539a2

14. Yang Y, Zhou H, Zhang L. Response to Letter to the Editor: Efficacy and Safety of Sintilimab Plus Pemetrexed and Platinum as First-Line Treatment for Locally Advanced or Metastatic Nonsquamous NSCLC: A Randomized, Double-Blind, Phase 3 Study (ORIENT-11). J Thorac Oncol 2020;15:e191-2.

15. Jiang H, Zheng Y, Qian J, et al. Efficacy and safety of sintilimab in combination with chemotherapy in previously untreated advanced or metastatic nonsquamous or squamous NSCLC: two cohorts of an open-label, phase 1b study. Cancer Immunol Immunother 2021;70:857-68.

16. Kato K, Shah MA, Enzinger P, et al. KEYNOTE-590: Phase III study of first-line chemotherapy with or without pembrolizumab for advanced esophageal cancer. Future Oncol 2019;15:1057-66.

17. Duan X, Zhang H, Zhou L, et al. Complete response to the combination of sintilimab and IBI305 for a patient with $\mathrm{HBV}$-associated hepatocellular carcinoma with multiple lung metastasis. Dig Liver Dis 2020;52:794-6.

18. Du Y, Si L, Mao L, et al. Efficacy of axitinib plus sintilimab in intermediate-and high-risk advanced renal cell carcinoma. Chinese Journal of Clinical Oncology 2020,47:513-6.

19. Gadgeel S, Rodríguez-Abreu D, Speranza G, et al. Updated Analysis From KEYNOTE-189: Pembrolizumab or Placebo Plus Pemetrexed and Platinum for Previously Untreated Metastatic Nonsquamous Non-Small-Cell Lung Cancer. J Clin Oncol 2020;38:1505-17.

20. Paz-Ares L, Vicente D, Tafreshi A, et al. A Randomized, Placebo-Controlled Trial of Pembrolizumab Plus Chemotherapy in Patients With Metastatic Squamous NSCLC: Protocol-Specified Final Analysis of KEYNOTE-407. J Thorac Oncol 2020;15:1657-69.

21. Jotte R, Cappuzzo F, Vynnychenko I, et al. Atezolizumab in Combination With Carboplatin and Nab-Paclitaxel in Advanced Squamous NSCLC (IMpower131): Results From a Randomized Phase III Trial. J Thorac Oncol 2020;15:1351-60.

22. Pietrantonio F, Randon G, Di Bartolomeo M, et al. Predictive role of microsatellite instability for PD-1 blockade in patients with advanced gastric cancer: a meta-analysis of randomized clinical trials. ESMO Open 2021;6:100036.

23. Xu J, Jiang H, Pan Y, et al. LBA53 Sintilimab plus 
chemotherapy (chemo) versus chemo as first-line treatment for advanced gastric or gastroesophageal junction (G/ GEJ) adenocarcinoma (ORIENT-16): First results of a randomized, double-blind, phase III study. Ann Oncol 2021;32:S1331.

24. Lam VK, Forde PM. Another Brick in the Wall: Sintilimab Plus Chemotherapy in Advanced Lung Cancer. J Thorac Oncol 2020;15:1556-8.

25. Jiang H, Zheng Y, Qian J, et al. Safety and efficacy of sintilimab combined with oxaliplatin/capecitabine as first-line treatment in patients with locally advanced or metastatic gastric/gastroesophageal junction adenocarcinoma in a phase Ib clinical trial. BMC Cancer 2020;20:760.

26. Zhang L, Mai W, Jiang W, et al. Sintilimab: A Promising

Cite this article as: Huang $\mathrm{N}$, Zhao C, Hu X, Zhang C, Xiong F, Huang W, Da L, Shen Y, Wu H. Safety and efficacy of sintilimab combination therapy for the treatment of 48 patients with advanced malignant tumors. Transl Cancer Res 2022;11(1):252-261. doi: 10.21037/tcr-22-54
Anti-Tumor PD-1 Antibody. Front Oncol 2020;10:594558.

27. Antonia SJ, Borghaei H, Ramalingam SS, et al. Four-year survival with nivolumab in patients with previously treated advanced non-small-cell lung cancer: a pooled analysis. Lancet Oncol 2019;20:1395-408.

28. Mok TSK, Wu YL, Kudaba I, et al. Pembrolizumab versus chemotherapy for previously untreated, PD-L1expressing, locally advanced or metastatic non-small-cell lung cancer (KEYNOTE-042): a randomised, open-label, controlled, phase 3 trial. Lancet 2019;393:1819-30.

29. Herbst RS, Baas P, Kim DW, et al. Pembrolizumab versus docetaxel for previously treated, PD-L1-positive, advanced non-small-cell lung cancer (KEYNOTE-010): a randomised controlled trial. Lancet 2016;387:1540-50. 